\title{
The nucleotide exchange factors of Hsp70 molecular chaperones
}

\author{
Andreas Bracher* and Jacob Verghese \\ Department of Cellular Biochemistry, Max-Planck-Institute of Biochemistry, Martinsried, Germany
}

Molecular chaperones of the Hsp70 family form an important hub in the cellular protein folding networks in bacteria and eukaryotes, connecting translation with the downstream machineries of protein folding and degradation. The Hsp70 folding cycle is driven by two types of cochaperones: J-domain proteins stimulate ATP hydrolysis by Hsp70, while nucleotide exchange factors (NEFs) promote replacement of Hsp70-bound ADP with ATP. Bacteria and organelles of bacterial origin have only one known NEF type for Hsp70, GrpE. In contrast, a large diversity of Hsp70 NEFs has been discovered in the eukaryotic cell. These NEFs belong to the Hsp110/Grp170, HspBP1/Sil1, and BAG domain protein families. In this short review we compare the structures and molecular mechanisms of nucleotide exchange factors for Hsp70 and discuss how these cochaperones contribute to protein folding and quality control in the cell.

Edited by:

Matthias Peter Mayer, Ruprecht-Karls-Universität Heidelberg, Germany

Reviewed by: Jason C. Young, McGill University, Canada

Rui Joaquim Sousa, University of Texas Health Science Center, San Antonio, USA

${ }^{*}$ Correspondence: Andreas Bracher Department of Cellular Biochemistry, Max-Planck-Institute of Biochemistry, Am Klopferspitz 18, 82152

Martinsried, Germany bracher@biochem.mpg.de

Specialty section: This article was submitted to Protein Folding, Misfolding and Degradation, a section of the journal Frontiers in Molecular Biosciences

Received: 13 February 2015 Accepted: 18 March 2015 Published: 07 April 2015

Citation: Bracher A and Verghese $J$ (2015) The nucleotide exchange factors of $\mathrm{Hsp} 70$ molecular chaperones.

Front. Mol. Biosci. 2:10. doi: 10.3389/fmolb.2015.00010

\section{Keywords: BAG domain, cochaperone, GrpE, HspBP1/Sil1, Hsp110/Grp170, protein folding, proteostasis}

\section{Introduction}

Molecular chaperones of the Hsp70 family use a nucleotide-dependent conformational cycle to support protein folding. Hsp70 proteins comprise an N-terminal nucleotide binding domain (NBD) and a substrate binding domain (SBD), which communicate by allosteric signals (reviewed in Mayer, 2013). The nucleotide is bound at the center of a two-lobed structure, commonly divided into subdomains IA, IB, IIA, and IIB. ATP binding favors a twist between the NBD lobes which allows extensive contacts with the SBD. In this conformation interactions with misfolded proteins are dynamic. In contrast, the ADP-bound state exhibits an extended structure with tight interactions between the SBD of Hsp70 and exposed hydrophobic peptide segments. ATP hydrolysis by Hsp70 thus induces stable substrate complex formation, and ATP re-binding triggers substrate release from Hsp70. Hsp70 has however high affinity for ADP and its intrinsic ATP hydrolase activity is low. Hence Hsp70 function critically depends on cochaperones, specifically J-domain proteins (JDP) and nucleotide exchange factors (NEFs), which accelerate ATP hydrolysis and ADP-ATP exchange, respectively. The great diversity of JDPs, especially in eukaryotes, suggests that Hsp70 is recruited for specific tasks by forming ternary complexes with substrates and these cochaperones. Eubacterial genomes encode only one NEF for Hsp70, GrpE. While in mitochondria and chloroplasts GrpE homologs are preserved, the cytosol and endoplasmic reticulum (ER) of eukaryotes contain three divergent families of NEFs: Hsp110/Grp170, HspBP1/Sil1 homologs and BAG-domain proteins (Höhfeld and Jentsch, 1997; Kabani et al., 2002; Steel et al., 2004; Dragovic et al., 2006; Raviol et al., 2006). The human genome encodes four Hsp110/Grp170 and two HspBP1/Sil1 homologs in addition to five BAG-domain proteins and two mitochondrial GrpE species (Figure 1). In addition, isoforms of NEFs resulting from alternative initiation sites and splicing exist. This NEF diversity may contribute to the appropriate allocation of Hsp70 folding capacity within the proteostasis network. Interestingly, metazoans, plants and some protists also 


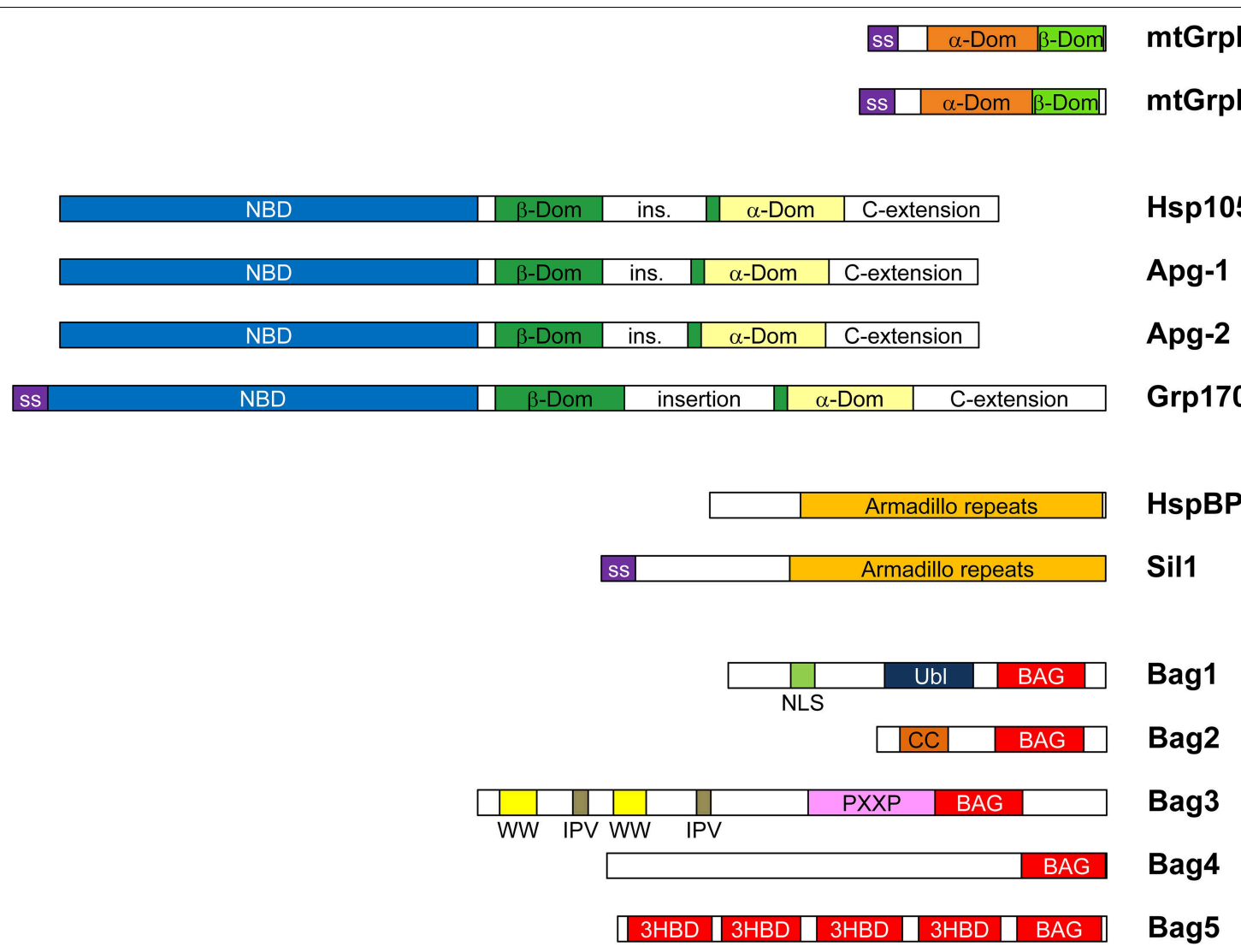

FIGURE 1 | Domain compositions of human NEF homologs. Human cells express two mitochondrial GrpE isoforms (mtGrpE1/GRPEL1 and mtGrpE2/GRPEL2), three Hsp110 homologs (Hsp105/HSPH1,

Apg-1/HSPA4L, and Apg-2/HSPA4-the codes designate the gene names) and five BAG-domain proteins. There is only one form of Grp170/HYOU1, HspBP1, and Sil1, respectively. Isoforms arising from alternative initiation sites and splicing were described for Hsp105, HspBP1, and Bag1 (not shown). mtGrpE isoforms contain mitochondrial signal sequences (ss). The $\alpha$ - and $\beta$-domains (orange and green, respectively) are conserved with GrpE from E. coli. The Hsp110/Grp170 family proteins consist of an N-terminal nucleotide binding domain (NBD, blue), a $\beta$-sandwich ( $\beta$-Dom, green) and a $\alpha$-helix bundle domain ( $\alpha$-Dom, pale yellow). All isoforms contain long variable insertions in the $\beta$-sandwich and at the C-terminus. SS indicates signal sequences for ER import of Grp170 and Sil1. HspBP1 and Sil have characteristic Armadillo repeat folds (orange). All members of the BAG family in humans, Bag1-5, contain C-terminal Hsp70-binding BAG domains (red), but have otherwise divergent domain composition. Bag1 contains an Ubiquitin-like domain (Ubl, dark blue), which might associate with the regulatory particle of the $26 \mathrm{~S}$ proteasome, and a NLS sequences (purple) for nuclear targeting. Bag2 contains a coiled-coil dimerization domain (CC, orange) (Page et al., 2012). Bag3 comprises multiple N-terminal sequence motifs including WW domains (WW, yellow), IPV sequence motifs (brown) and PXXP repeats (pink), which mediate interactions with proline-rich motifs, HspB8 and SH3 domains, respectively (Doong et al., 2000; Fuchs et al., 2010; Iwasaki et al., 2010; Ulbricht et al., 2013). Bag5 has four additional 3-helix bundle domains of unknown function (Arakawa et al., 2010). Bag6 is not shown because the original assignment as an NEF of Hsp70 was incorrect (Mock et al., 2015). harbor the additional cochaperone Hip (gene ST13), which antagonizes NEF function by stalling Hsp70 cycling and stabilizing Hsp70 complexes with specific substrates.

In this review we compare the structures and molecular mechanisms of different NEF families and discuss how these cochaperones contribute to protein remodeling, folding and quality control in the cell. For a more comprehensive overview, please see our earlier work (Bracher and Verghese, 2015).

\section{GrpE, the Bacterial NEF}

Protein folding by the eubacterial Hsp70 homolog DnaK in Escherichia coli depends on GrpE, which is encoded by an essential gene (Ang and Georgopoulos, 1989). Together with the JDP
DnaJ, GrpE greatly accelerates ATP hydrolysis and thus conformational cycling of DnaK (Liberek et al., 1991; Laufen et al., 1999). However, it should be noted that E. coli also expresses two specialized Hsp70 isoforms, HscA and $\mathrm{HscC}$, which do not depend on GrpE (Brehmer et al., 2001).

Structurally, GrpE is composed of an $\alpha$-helical dimerization domain and a $\beta$-domain that mediates most of the interactions with DnaK (Harrison et al., 1997). The $\alpha$-helical domains form a stalk-like, coiled-coil structure with a four-helix bundle at the $\mathrm{C}$-terminal end; the $\beta$-domains protrude like wings from the helix bundle (Figure 2). In the complex with the NBD of DnaK, one $\beta$-domain inserts into the nucleotide binding cleft, forcing the nucleotide binding pocket open by rotation of subdomain IIB (Figure 2). This NBD conformation has greatly 


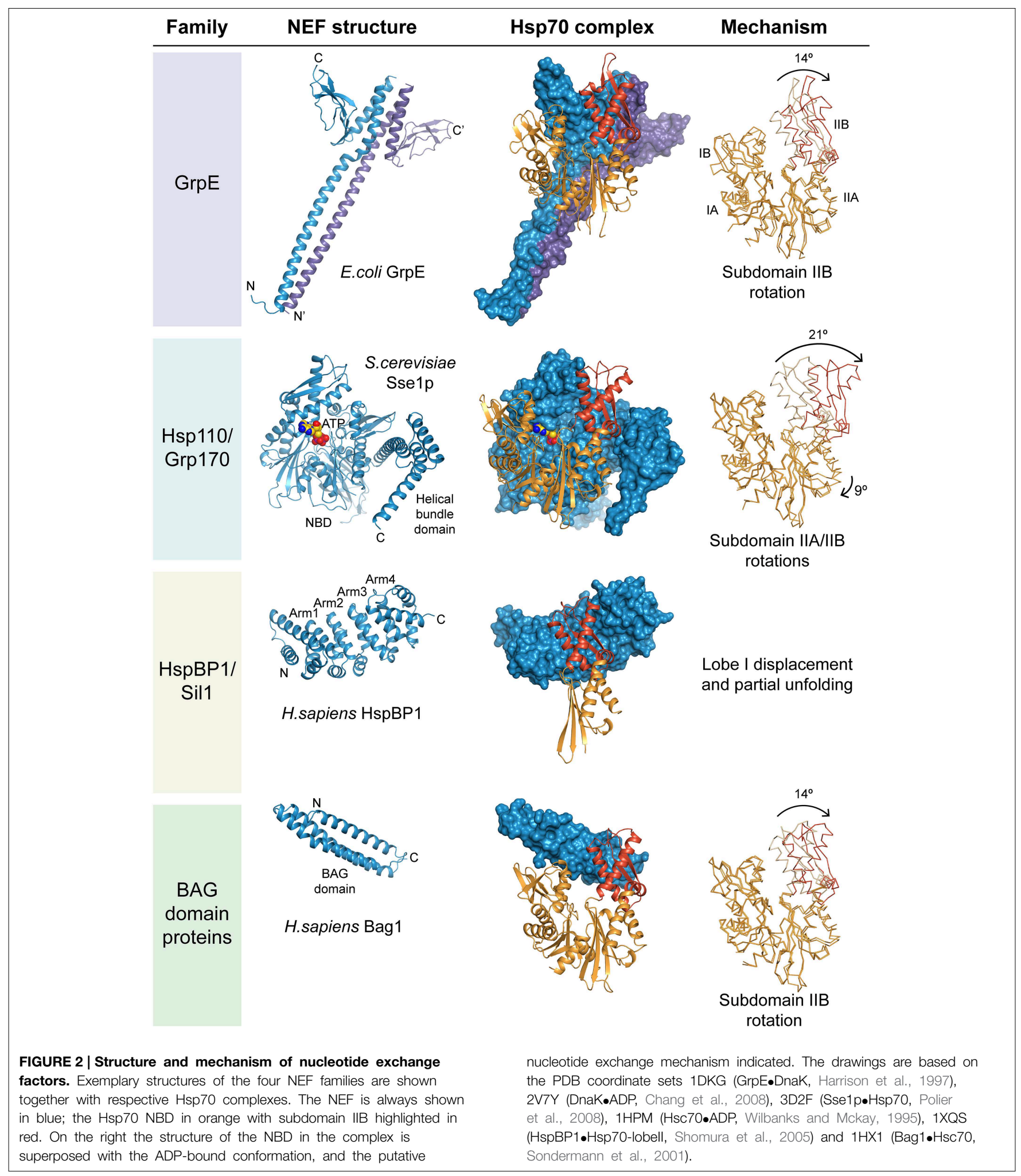

diminished affinity for nucleotide. Simulations of the highly conserved NBD suggest that subdomain IIB motion is facilitated by a flexible hinge connection (Ung et al., 2013). GrpE thus utilizes an in-built feature of Hsp70 for its function. In addition to the $\beta$-domain, parts of the stalk and flexible $\mathrm{N}$-terminus of GrpE contribute to DnaK binding. The latter segment appears 
to compete with substrate binding to DnaK (Harrison et al., 1997).

GrpE function in E. coli appears to be regulated by temperature (Grimshaw et al., 2001). At the optimal growth temperature, GrpE is dimeric and active, enabling rapid DnaK cycling needed for folding newly synthesized proteins. Under thermal stress, the stalk of GrpE appears to unfold, which prevents the cochaperone from binding to DnaK•substrate complexes and thus limits futile cycling and ATP expenditure. During thermal stress, interactions with DnaK stabilize substrates against aggregation. The transition in GrpE is fully reversible and the DnaK-DnaJGrpE system begins to refold DnaK-stabilized substrates upon recovery.

\section{Eukaryotic GrpE Homologs}

Mitochondria and chloroplasts, the eukaryotic organelles of eubacterial origin preserve the bacterial Hsp70 system including GrpE homologs. The most conspicuous function of this system is the import of organellar proteins from the cytosol across membranes (see accompanying reviews). This is essential because most of the matrix/stroma proteins are encoded in the nucleus. Moreover, protein folding within these organelles also depends on GrpE function. The thermosensory function of GrpE appears to be preserved in eukaryotes (Moro and Muga, 2006; Willmund et al., 2007).

\section{Evolution of Eukaryotic NEFs}

In contrast to DnaK, authentic eukaryotic Hsp70 homologs have weakened contacts across the nucleotide binding cleft. Probably the function of the eukaryotic Hsp70 ancestor did not require NEFs. Dependence on NEFs apparently evolved only later, and thus modern GrpE and the mammalian NEF Bag1 function only with their cognate Hsp70 partners (Brehmer et al., 2001). In the presence of physiological phosphate concentrations, the ADP dissociation rates of eukaryotic Hsp70 diminish to the levels similar to DnaK in E. coli, and thus NEFs are required for efficient cycling in vivo (Gässler et al., 2001; Arakawa et al., 2011). The most ancient and universal type of eukaryotic NEF is Hsp110/Grp170, which shares its molecular architecture with canonical Hsp70 proteins (Figure 2). The other two NEF families, HspBP1/Sil1 homologs and BAGdomain proteins, have generic structural scaffolds, Armadillo repeats and three-helix bundles, respectively, which are also found in other functional contexts such as nuclear transport and vesicle fusion (Figure 2). Interestingly, despite their considerable structural diversity, all NEFs target subdomain IIB in the NBD of Hsp70. This suggests convergent evolution toward activating the molecular switch built into the Hsp70 NBD. It appears that the structurally homologous NEFs HspBP1 and Sillp evolved independently, since their contact regions with the cognate Hsp70 homolog differ markedly (Shomura et al., 2005; Yan et al., 2011). Convergent evolution might also explain the perplexing diversity in BAG-domain architecture, which became only apparent when the individual structures were determined.

\section{Nucleotide Exchange Mechanism}

All eukaryotic NEFs seem to capture open conformations of the NBD. GrpE literally drives a molecular wedge into the nucleotide binding cleft of DnaK, whereas the Hsp110 homolog in Saccharomyces cerevisiae, Sse1p, attaches to the flank of subdomain IIB while anchoring itself onto the remainder of the NBD (Polier et al., 2008). HspBP1/Sil1 homologs and BAG-domain proteins use their bulk mass to fix Hsp70 in an open conformation. HspBP1 binding even induces partial unfolding of the NBD. Because their shapes vary considerably, the NBD subdomains are displaced in different ways, as evidenced by cocrystal structures with Hsp70 NBDs (Figure 2). ATP but not ADP binding subsequently displaces NEFs from eukaryotic Hsp70 for a new round of substrate binding.

\section{Hsp110/Grp170}

Hsp110 and its ER-lumenal homolog Grp170 share their domain architecture with canonical Hsp70 proteins, consisting of an $\mathrm{N}$ terminal NBD, followed by a $\beta$-sandwich and an $\alpha$-helix bundle domain, but have long insertions and C-terminal extensions compared to Hsp70 (Figure 1). Their sequence conservation is much lower than in canonical Hsp70. Crystal structures of the Hsp110 homolog Sse1p in complex with Hsp70 showed that the two NBDs face each other (Figure 2) (Polier et al., 2008; Schuermann et al., 2008). The NBD of Hsp70 is fixed in an open conformation by additional contacts with the $\alpha$-helix bundle domain of Sse1p. These contacts are highly conserved in the Hsp110/Grp170 family, and presumably all members employ the same binding mode (Andreasson et al., 2010; Hale et al., 2010). To function, Sse1p requires ATP binding, which induces a compact conformation, but not ATP hydrolysis (Shaner et al., 2004; Raviol et al., 2006). Indeed, expression of ATPbinding competent, but ATPase-deficient Sse1p mutants rescues the lethal phenotype of the deletion of SSE1/SSE2. Because of low sequence conservation in the $\beta$-sandwich domain, it is unclear whether the substrate binding mode seen in DnaK.ADP is also employed by Hsp110/Grp170. Hsp110 homologs seem to prefer aromatic residues in target sequences, while canonical Hsp70 has a bias toward aliphatic hydrophobic and proline residues (Xu et al., 2012). Mammalian and yeast Hsp 110 homologs are potent holdases for misfolded luciferase, preventing its aggregation until refolding with Hsp70/Hsp40 commences (Oh et al., 1997, 1999). Sselp requires heat activation for this activity (Polier et al., 2010). Whether nucleotide-dependent cycling of Hsp110/Grp170 is required for holdase activity is controversial.

\section{HspBP1/Sil1}

HspBP1 and Sil1 represent the cytosolic and ER-lumenal forms of a NEF family with Armadillo repeat architecture, respectively. Sill homologs can be identified in most eukaryotes; Caenorhabditis elegans apparently lacks a cytosolic HspBP1 homolog. The C-terminal 260-residue NEF domain of human HspBP1 consists of four Armadillo repeats capped at each end with $\alpha$-helix pairs. The short $\mathrm{N}$-terminal regions are highly 
divergent and their function is unknown. The structure of the human HspBP1 NEF domain in complex with a fragment of Hsp70 showed that the concave face of the Armadillo repeat structure wraps around subdomain IIB of the NBD (Figure 2) (Shomura et al., 2005). Comparison with other structures of the Hsp70 NBD suggests that the bulk of HspBP1 would clash with subdomain IB. Protease sensitivity and tryptophan fluorescence indicates that Hsp70 evades this through local unfolding in the complex. Surprisingly, the yeast ER paralog Sillp employs different contacts and stops at mere opening of the nucleotide binding cleft (Yan et al., 2011). Interestingly, the interactions of metazoan Sil1 with the ER-Hsp70, BiP, seem more similar to HspBP1 than to the putative yeast ortholog Sillp (Hale et al., 2010; Howes et al., 2012). Whether ATP binding preempts NBD unfolding under physiological conditions remains unknown.

\section{BAG Domain Proteins}

The BAG domain-containing proteins represent the most divergent group among eukaryotic NEFs. Human Bag1 was the first eukaryotic NEF to be identified, after its initial characterization as the binding partner of anti-apoptotic protein $\mathrm{Bcl}-2$ (Höhfeld and Jentsch, 1997). It contains a 3-helix bundle domain of 110 residues having NEF activity (Figure 2) (Sondermann et al., 2001). Subsequently four more homologs with putative Bag domains were identified in humans, Bag2-Bag5 (Takayama and Reed, 2001). All have distinct domain compositions (Figure 1). Structural analysis of the respective Bag domains revealed a surprising diversity of $\alpha$-helix bundle architectures, but all share a conserved sequence signature that targets subdomain IIB of Hsp70. The Hsp70-binding BAG domains of Bag3, Bag4, and Bag5 form shorter 3-helix bundles than Bag1 (Briknarova et al., 2002; Brockmann et al., 2004; Arakawa et al., 2010). The "Brand New Bag" (BNB) domain of Bag2 has a dimeric four-helix bundle structure that can accommodate two Hsp70s (Xu et al., 2008). This could help target complexes of multiple Hsp70 molecules attached to one substrate molecule.

The diverse domains found together with the NEF domain in BAG proteins probably allow recruitment of Hsp70 for specific purposes. However, only the domain compositions of Bag1 and Bag3 appear conserved among metazoans. Bag1 homologs also seem to occur in plants (Kabbage and Dickman, 2008). Bag1 contains an Ubiquitin-like domain (Ubl) in addition to the NEF domain, suggesting a role in targeting substrates for proteasomal degradation. In murine Bag1, the Ubl domain also mediates interactions with EGF-like growth factor (Hung et al., 2014). Bag3 has multiple interaction motifs and connects Hsc70 with the small heat shock protein HspB8 and the dynein adaptor protein $14-3-3 \gamma$ in targeting protein aggregates for degradation by autophagy via the microtubule network (Arndt et al., 2010; Fuchs et al., 2010; Xu et al., 2013). Bag2 forms stable ternary complexes with Hsc70 and the Hsp70-associated dimeric ubiquitin ligase CHIP, inhibiting proteasome targeting of inducible Hsp70 and substrate proteins (Arndt et al., 2005; Dai et al., 2005). The BNB domain has also been implicated in binding to substrate directly (Xu et al., 2008).

\section{Antagonism between Hip and NEF Function}

The dimeric multi-domain protein Hip antagonizes the function of Bag1 and probably other NEFs (Kanelakis et al., 2000). Hip slows the dissociation of ADP from Hsp70, thus stabilizing substrate protein association in presence of ATP (Höhfeld et al., 1995; Li et al., 2013). The crystal structure of the core complex of Hip with Hsp70.ADP revealed that the binding interfaces of Hip and NEFs overlap, resulting in mutually exclusive binding (Li et al., 2013). The binding affinity of NEFs - with the exception of Bag $2-$ is however $\sim 100$ times higher, and stable binding of Hip thus requires additional interactions. Simultaneous interactions with two Hsp70 molecules attached to a slow-folding substrate or an aggregate would boost affinity toward the Hip dimer through avidity. Hip might also directly recognize specific substrates such as the Hsp90-client protein glucocorticoid receptor via its DP domains (Nelson et al., 2004). Interaction of Hsp70substrate complexes with Hip might thus enable slow cycling and limit futile energy expenditure by Hsp70 on ill-fated substrate proteins and downstream chaperone clients. Concomitantly, Hip binding might also prolong the time window for proteasomal targeting, consistent with increased disposal of mutant androgen receptor upon Hip overexpression (Wang et al., 2013).

\section{Involvement of NEFs in Protein Folding and Import}

In the model organism S. cerevisiae, Sse1p is by far the most abundant cytosolic NEF, followed by its inducible isoform Sse2p and the HspBP1 ortholog Fes1p. Together they constitute about 1/10 of the total concentration of cellular Hsp70 (Ghaemmaghami et al., 2003; Kulak et al., 2014). The Bag protein Snllp is only present at low concentration and probably highly specialized (Verghese and Morano, 2012). Sse1p collaborates with ribosomebound and cytosolic Hsp70 isoforms in folding and processing a large proportion of newly made proteins (Yam et al., 2005). Deletion of SSE1 causes a growth defect, which is only partially rescued by Fes1p overexpression (Raviol et al., 2006). Overexpression of Sse1p impairs growth as well, suggesting that Sse1p competes with ATP binding to Hsp70 under these conditions (Liu et al., 1999). Well-balanced concentration ratios between Hsp70 and NEFs seem essential for proper protein folding. Sse1p, Sse2p, and Fes1p are upregulated together with Hsp70 under heat stress. Deletion of FES1 causes a massive heat shock response in the absence of thermal stress (Gowda et al., 2013; Abrams et al., 2014), a folding defect of the reporter protein firefly luciferase (FLuc) (Shomura et al., 2005) and a thermosensitivity phenotype (Ahner et al., 2005). Besides their function in de novo protein folding, Sse1p and Fes1p also contribute in distributing substrates to downstream chaperones Hsp90 and TRiC and to clear misfolded species (Goeckeler et al., 2002; Mcclellan et al., 2005; Gowda et al., 2013).

The diversity of eukaryotic NEFs might enable adaptation of the Hsp70 cycling rate to the folding needs of specific substrate proteins. Consistent with this hypothesis, comparative in vitro studies with mammalian homologs showed that certain Hsp70/JDP/NEF combinations work much better in FLuc folding 
than others and some not at all (Tzankov et al., 2008; Rauch and Gestwicki, 2014). How specific combinations select suitable clients is unknown. NEF diversity might also allow differential distribution of substrates to downstream chaperones via adaptor proteins like HOP (Knapp et al., 2014). Functional redundancy of mammalian NEFs is, however, considerable. While knockout of the Hsp110 isoform Hsp105 causes no obvious defect (Nakamura et al., 2008), individual deletions of HspBP1 and Apg-2, another Hsp110 isoform, severely affect spermatogenesis (Held et al., 2011; Rogon et al., 2014). Only simultaneous knockout of Apg-1 and Apg-2 is lethal (Mohamed et al., 2014).

The ER-lumenal NEFs Sill and Grp170 contribute substantially to protein folding in the secretory pathway (Behnke et al., 2015). In addition they function together with BiP and the poreassociated JDP Sec63 in protein import, possibly by preventing substrate backsliding through $\mathrm{BiP}$ binding and release cycles (Zimmermann et al., 2011). Deletions of the respective NEF homologs in yeast, Sillp and Lhs1p, activate the Unfolded Protein Response and cause import defects (Tyson and Stirling, 2000). Both Sil1 and Grp170 are upregulated under ER stress. In mice, knockout of Grp170 is lethal (Kitao et al., 2004). Mutations that inactivate Sill cause Marinesco-Sjögren syndrome in humans (Anttonen et al., 2005; Senderek et al., 2005) and the Woozy phenotype in mice (Zhao et al., 2005), respectively, conditions characterized by neurodegeneration and myopathy, likely because persistent ER stress induces apoptosis.

\section{Role in Protein Quality Control}

The folding of Cystic Fibrosis Transmembrane Conductance Regulator (CFTR) may serve as a paradigm for the role of NEFs in protein quality control involving proteasomal degradation. CFTR, a transmembrane protein, has inefficiently folding cytoplasmic domains, which may explain why a large proportion undergoes CHIP-mediated degradation before reaching the epithelial membrane (Meacham et al., 2001). Hsp105 appears to have a prominent role in CFTR folding at the ER and later at the epithelial membrane, employing its holdase activity (Saxena et al., 2012). Binding of HspBP1 to Hsc70 stimulates CFTR maturation (Alberti et al., 2004), whereas Bag1 collaborates with CHIP in CFTR degradation (Demand et al., 2001).

The alternative to proteasomal degradation is disposal of faulty proteins by the lysosome via autophagy. This route seems especially important for muscle structure maintenance, which requires the adaptor function of Bag3 (Arndt et al., 2010; Ulbricht et al., 2013). Bag3 deletion in mice results in severe myopathy (Homma et al., 2006). Similar phenotypes were reported for deletions of the probable Drosophila melanogaster and C. elegans

\section{References}

Abrams, J. L., Verghese, J., Gibney, P. A., and Morano, K. A. (2014). Hierarchical functional specificity of cytosolic heat shock protein 70 (Hsp70) nucleotide exchange factors in yeast. J. Biol. Chem. 289, 13155-13167. doi: 10.1074/jbc.M113.530014 orthologs, Starvin, and unc23, respectively (Arndt et al., 2010; Papsdorf et al., 2014). Bag3 mutations in humans are associated with autosomal dominant forms of myofibrillar myopathy and dilated cardiomyopathy (Selcen et al., 2009; Norton et al., 2011). Interestingly, Bag3 is the only stress-inducible BAG-domain protein (Franceschelli et al., 2008), and its increased abundance might tip the balance from proteasomal to lysosomal degradation (Gamerdinger et al., 2009).

Hsp110 proteins were found associated with aggregates of misfolding-prone proteins that cause neurodegenerative disease, including mutant SOD in Amyotrophic Lateral Sclerosis (Wang et al., 2009) and poly-Q androgen receptor in Spinal and Bulbar Muscular Atrophy (Ishihara et al., 2003). Hsp105-knockout mice accumulate hyper-phosphorylated tau similar to neurofibrillary tangles in Alzheimer's disease (Eroglu et al., 2010). Hsp110, together with Hsp70 and Hsp40, has been implicated in a metazoan disaggregase activity analogous to $\mathrm{ClpB} / \mathrm{Hsp} 104$ in bacteria and fungi, which might resolubilize such aggregates for new folding attempts or proteasomal degradation (Shorter, 2011; Rampelt et al., 2012). Hsp110 thus may be considered the ultimate profolding NEF in eukaryotes, consistent with increased vulnerability of fast-growing cancer cells with a dominant-negative allele of this cochaperone (Dorard et al., 2011).

\section{Outlook}

How the different components in the Hsp70 system intersect with other branches of the proteostasis network is only beginning to emerge. Different expression levels of competing NEFs and opposing factors like Hip may change the fate of specific substrate proteins in individual cell types. The intracellular distribution of proteostasis components might furthermore alter the dynamics of protein folding and degradation. Thus, information on cellular dose and distribution in healthy and diseased cells will be needed for an integrated picture of NEF roles in Hsp70-dependent cellular processes.

\section{Author Contributions}

Both authors contributed to drafting and critically revising the work.

\section{Acknowledgments}

We thank F. Ulrich Hartl and the Max Planck Society for continuous support, and Courtney L. Klaips for critical reading of the manuscript. JV is supported by the Rudolf Haas Fellowship from the Jung Foundation for Science and Research. functions of Hsp70, Hsp90, and an Hsp70 nucleotide exchange factor during protein biogenesis in yeast. Arch. Biochem. Biophys. 435, 32-41. doi: 10.1016/j.abb.2004.11.005

Alberti, S., Bohse, K., Arndt, V., Schmitz, A., and Höhfeld, J. (2004). The Co-Chaperone HspBP1 Inhibits the CHIP ubiquitin ligase and stimulates the 
maturation of the cystic fibrosis transmembrane conductance regulator. Mol. Biol. Cell 15, 4003-4010. doi: 10.1091/mbc.E04-04-0293

Andreasson, C., Rampelt, H., Fiaux, J., Druffel-Augustin, S., and Bukau, B. (2010). The endoplasmic reticulum Grp170 acts as a nucleotide exchange factor of Hsp70 via a mechanism similar to that of the cytosolic Hsp110. J. Biol. Chem. 285, 12445-12453. doi: 10.1074/jbc.M109.096735

Ang, D., and Georgopoulos, C. (1989). The heat-shock-regulated grpE gene of Escherichia coli is required for bacterial growth at all temperatures but is dispensable in certain mutant backgrounds. J. Bacteriol. 171, 2748-2755.

Anttonen, A. K., Mahjneh, I., Hamalainen, R. H., Lagier-Tourenne, C., Kopra, O., Waris, L., et al. (2005). The gene disrupted in Marinesco-Sjogren syndrome encodes SIL1, an HSPA5 cochaperone. Nat. Genet. 37, 1309-1311. doi: $10.1038 /$ ng 1677

Arakawa, A., Handa, N., Ohsawa, N., Shida, M., Kigawa, T., Hayashi, F., et al. (2010). The C-terminal BAG domain of BAG5 induces conformational changes of the Hsp70 nucleotide-binding domain for ADP-ATP exchange. Structure 18, 309-319. doi: 10.1016/j.str.2010.01.004

Arakawa, A., Handa, N., Shirouzu, M., and Yokoyama, S. (2011). Biochemical and structural studies on the high affinity of Hsp70 for ADP. Protein Sci. 20, 1367-1379. doi: 10.1002/pro.663

Arndt, V., Daniel, C., Nastainczyk, W., Alberti, S., and Höhfeld, J. (2005). BAG2 acts as an inhibitor of the chaperone-associated ubiquitin ligase CHIP. Mol. Biol. Cell 16, 5891-5900. doi: 10.1091/mbc.E05-07-0660

Arndt, V., Dick, N., Tawo, R., Dreiseidler, M., Wenzel, D., Hesse, M., et al. (2010). Chaperone-assisted selective autophagy is essential for muscle maintenance. Curr. Biol. 20, 143-148. doi: 10.1016/j.cub.2009.11.022

Behnke, J., Feige, M. J., and Hendershot, L. M. (2015). BiP and its nucleotide exchange factors Grp170 and Sil1: mechanisms of action and biological functions. J. Mol. Biol. 427, 1589-1608. doi: 10.1016/j.jmb.2015.02.011

Bracher, A., and Verghese, J. (2015). GrpE, Hsp110/Grp170, HspBP1/Sill and BAG Domain Proteins: Nucleotide Exchange Factors for Hsp70 Molecular Chaperones. Subcell. Biochem. 78, 1-33. doi: 10.1007/978-3-319-11731-7_1

Brehmer, D., Rüdiger, S., Gässler, C. S., Klostermeier, D., Packschies, L., Reinstein, J., et al. (2001). Tuning of chaperone activity of Hsp70 proteins by modulation of nucleotide exchange. Nat. Struct. Biol. 8, 427-432. doi: 10.1038/87588

Briknarova, K., Takayama, S., Homma, S., Baker, K., Cabezas, E., Hoyt, D. W., et al. (2002). BAG4/SODD protein contains a short BAG domain. J. Biol. Chem. 277, 31172-31178. doi: 10.1074/jbc.M202792200

Brockmann, C., Leitner, D., Labudde, D., Diehl, A., Sievert, V., Büssow, K., et al. (2004). The solution structure of the SODD BAG domain reveals additional electrostatic interactions in the HSP70 complexes of SODD subfamily BAG domains. FEBS Lett. 558, 101-106. doi: 10.1016/S0014-5793(03)01490-X

Chang, Y. W., Sun, Y. J., Wang, C., and Hsiao, C. D. (2008). Crystal structures of the $70-\mathrm{kDa}$ heat shock proteins in domain disjoining conformation. J. Biol. Chem. 283, 15502-15511. doi: 10.1074/jbc.M708992200

Dai, Q., Qian, S. B., Li, H. H., Mcdonough, H., Borchers, C., Huang, D., et al. (2005). Regulation of the cytoplasmic quality control protein degradation pathway by BAG2. J. Biol. Chem. 280, 38673-38681. doi: 10.1074/jbc.M507986200

Demand, J., Alberti, S., Patterson, C., and Höhfeld, J. (2001). Cooperation of a ubiquitin domain protein and an E3 ubiquitin ligase during chaperone/proteasome coupling. Curr. Biol. 11, 1569-1577. doi: 10.1016/S0960-9822(01)00487-0

Doong, H., Price, J., Kim, Y. S., Gasbarre, C., Probst, J., Liotta, L. A., et al. (2000). CAIR-1/BAG-3 forms an EGF-regulated ternary complex with phospholipase C-gamma and Hsp70/Hsc70. Oncogene 19, 4385-4395. doi: 10.1038/sj.onc. 1203797

Dorard, C., De Thonel, A., Collura, A., Marisa, L., Svrcek, M., Lagrange, A., et al. (2011). Expression of a mutant HSP110 sensitizes colorectal cancer cells to chemotherapy and improves disease prognosis. Nat. Med. 17, 1283-1289. doi: $10.1038 / \mathrm{nm} .2457$

Dragovic, Z., Broadley, S. A., Shomura, Y., Bracher, A., and Hartl, F. U. (2006). Molecular chaperones of the Hsp110 family act as nucleotide exchange factors of Hsp70s. EMBO J. 25, 2519-2528. doi: 10.1038/sj.emboj.7601138

Eroglu, B., Moskophidis, D., and Mivechi, N. F. (2010). Loss of Hsp110 leads to age-dependent tau hyperphosphorylation and early accumulation of insoluble amyloid beta. Mol. Cell. Biol. 30, 4626-4643. doi: 10.1128/MCB.01493-09

Franceschelli, S., Rosati, A., Lerose, R., De Nicola, S., Turco, M. C., and Pascale, M. (2008). Bag3 gene expression is regulated by heat shock factor 1. J. Cell Physiol. 215, 575-577. doi: 10.1002/jcp.21397
Fuchs, M., Poirier, D. J., Seguin, S. J., Lambert, H., Carra, S., Charette, S. J., et al. (2010). Identification of the key structural motifs involved in HspB8/HspB6Bag3 interaction. Biochem. J. 425, 245-255. doi: 10.1042/BJ20090907

Gamerdinger, M., Hajieva, P., Kaya, A. M., Wolfrum, U., Hartl, F. U., and Behl, C. (2009). Protein quality control during aging involves recruitment of the macroautophagy pathway by BAG3. EMBO J. 28, 889-901. doi: 10.1038/emboj.2009.29

Gässler, C. S., Wiederkehr, T., Brehmer, D., Bukau, B., and Mayer, M. P. (2001). Bag-1M accelerates nucleotide release for human Hsc70 and Hsp70 and can act concentration-dependent as positive and negative cofactor. J. Biol. Chem. 276, 32538-32544. doi: 10.1074/jbc.M105328200

Ghaemmaghami, S., Huh, W. K., Bower, K., Howson, R. W., Belle, A., Dephoure, N., et al. (2003). Global analysis of protein expression in yeast. Nature 425, 737-741. doi: 10.1038/nature02046

Goeckeler, J. L., Stephens, A., Lee, P., Caplan, A. J., and Brodsky, J. L. (2002). Overexpression of yeast Hsp110 homolog Sse1p suppresses ydj1-151 thermosensitivity and restores Hsp90-dependent activity. Mol. Biol. Cell 13, 2760-2770. doi: 10.1091/mbc.02-04-0051

Gowda, N. K., Kandasamy, G., Froehlich, M. S., Dohmen, R. J., and Andreasson, C. (2013). Hsp70 nucleotide exchange factor Fes1 is essential for ubiquitindependent degradation of misfolded cytosolic proteins. Proc. Natl. Acad. Sci. U.S.A. 110, 5975-5980. doi: 10.1073/pnas.1216778110

Grimshaw, J. P., Jelesarov, I., Schonfeld, H. J., and Christen, P. (2001). Reversible thermal transition in GrpE, the nucleotide exchange factor of the DnaK heatshock system. J. Biol. Chem. 276, 6098-6104. doi: 10.1074/jbc.M009290200

Hale, S. J., Lovell, S. C., De Keyzer, J., and Stirling, C. J. (2010). Interactions between Kar2p and its nucleotide exchange factors Sillp and Lhs1p are mechanistically distinct. J. Biol. Chem. 285, 21600-21606. doi: 10.1074/jbc.M110.111211

Harrison, C. J., Hayer-Hartl, M., Di Liberto, M., Hartl, F., and Kuriyan, J. (1997). Crystal structure of the nucleotide exchange factor GrpE bound to the ATPase domain of the molecular chaperone DnaK. Science 276, 431-435. doi: $10.1126 /$ science.276.5311.431

Held, T., Barakat, A. Z., Mohamed, B. A., Paprotta, I., Meinhardt, A., Engel, W., et al. (2011). Heat-shock protein HSPA4 is required for progression of spermatogenesis. Reproduction 142, 133-144. doi: 10.1530/REP-11-0023

Höhfeld, J., and Jentsch, S. (1997). GrpE-like regulation of the hsc70 chaperone by the anti-apoptotic protein BAG-1. EMBO J. 16, 6209-6216. doi: 10.1093/emboj/16.20.6209

Höhfeld, J., Minami, Y., and Hartl, F. U. (1995). Hip, a novel cochaperone involved in the eukaryotic Hsc70/Hsp40 reaction cycle. Cell 83, 589-598. doi: 10.1016/0092-8674(95)90099-3

Homma, S., Iwasaki, M., Shelton, G. D., Engvall, E., Reed, J. C., and Takayama, S. (2006). BAG3 deficiency results in fulminant myopathy and early lethality. Am. J. Pathol. 169, 761-773. doi: 10.2353/ajpath.2006.060250

Howes, J., Shimizu, Y., Feige, M. J., and Hendershot, L. M. (2012). C-terminal mutations destabilize SIL1/BAP and can cause Marinesco-Sjogren syndrome. J. Biol. Chem. 287, 8552-8560. doi: 10.1074/jbc.M111.333286

Hung, K. W., Huang, H. W., Cho, C. C., Chang, S. C., and Yu, C. (2014). Nuclear magnetic resonance structure of the cytoplasmic tail of heparin binding EGF-like growth factor (proHB-EGF-CT) complexed with the ubiquitin homology domain of Bcl-2-associated athanogene 1 from Mus musculus (mBAG-1-UBH). Biochemistry 53, 1935-1946. doi: 10.1021/bi5003019

Ishihara, K., Yamagishi, N., Saito, Y., Adachi, H., Kobayashi, Y., Sobue, G., et al. (2003). Hsp105alpha suppresses the aggregation of truncated androgen receptor with expanded CAG repeats and cell toxicity. J. Biol. Chem. 278, 25143-25150. doi: 10.1074/jbc.M302975200

Iwasaki, M., Tanaka, R., Hishiya, A., Homma, S., Reed, J. C., and Takayama, S. (2010). BAG3 directly associates with guanine nucleotide exchange factor of Rap1, PDZGEF2, and regulates cell adhesion. Biochem. Biophys. Res. Commun. 400, 413-418. doi: 10.1016/j.bbrc.2010.08.092

Kabani, M., Beckerich, J. M., and Brodsky, J. L. (2002). Nucleotide exchange factor for the yeast Hsp70 molecular chaperone Ssalp. Mol. Cell. Biol. 22, 4677-4689. doi: 10.1128/MCB.22.13.4677-4689.2002

Kabbage, M., and Dickman, M. B. (2008). The BAG proteins: a ubiquitous family of chaperone regulators. Cell. Mol. Life Sci. 65, 1390-1402. doi: 10.1007/s00018008-7535-2

Kanelakis, K. C., Murphy, P. J., Galigniana, M. D., Morishima, Y., Takayama, S., Reed, J. C., et al. (2000). hsp70 interacting protein Hip does not affect 
glucocorticoid receptor folding by the hsp90-based chaperone machinery except to oppose the effect of BAG-1. Biochemistry 39, 14314-14321. doi: $10.1021 /$ bi001671c

Kitao, Y., Hashimoto, K., Matsuyama, T., Iso, H., Tamatani, T., Hori, O., et al. (2004). ORP150/HSP12A regulates Purkinje cell survival: a role for endoplasmic reticulum stress in cerebellar development. J. Neurosci. 24, 1486-1496. doi: 10.1523/JNEUROSCI.4029-03.2004

Knapp, R. T., Wong, M. J., Kollmannsberger, L. K., Gassen, N. C., Kretzschmar, A., Zschocke, J., et al. (2014). Hsp70 cochaperones HspBP1 and BAG-1M differentially regulate steroid hormone receptor function. PLOS ONE 9:e85415. doi: 10.1371/journal.pone.0085415

Kulak, N. A., Pichler, G., Paron, I., Nagaraj, N., and Mann, M. (2014). Minimal, encapsulated proteomic-sample processing applied to copy-number estimation in eukaryotic cells. Nat. Methods 11, 319-324. doi: 10.1038/nmeth. 2834

Laufen, T., Mayer, M. P., Beisel, C., Klostermeier, D., Mogk, A., Reinstein, J., et al. (1999). Mechanism of regulation of hsp70 chaperones by DnaJ cochaperones. Proc. Natl. Acad. Sci. U.S.A. 96, 5452-5457. doi: 10.1073/pnas.96. 10.5452

Li, Z., Hartl, F. U., and Bracher, A. (2013). Structure and function of Hip, an attenuator of the Hsp70 chaperone cycle. Nat. Struct. Mol. Biol. 20, 929-935. doi: $10.1038 / \mathrm{nsmb} .2608$

Liberek, K., Marszalek, J., Ang, D., Georgopoulos, C., and Zylicz, M. (1991). Escherichia coli DnaJ and GrpE heat shock proteins jointly stimulate ATPase activity of DnaK. Proc. Natl. Acad. Sci. U.S.A. 88, 2874-2878. doi: 10.1073/pnas.88.7.2874

Liu, X. D., Morano, K. A., and Thiele, D. J. (1999). The yeast Hsp110 family member, Sse1, is an Hsp90 cochaperone. J. Biol. Chem. 274, 26654-26660. doi: $10.1074 /$ jbc. 274.38 .26654

Mayer, M. P. (2013). Hsp70 chaperone dynamics and molecular mechanism. Trends Biochem. Sci. 38, 507-514. doi: 10.1016/j.tibs.2013.08.001

Mcclellan, A. J., Scott, M. D., and Frydman, J. (2005). Folding and quality control of the VHL tumor suppressor proceed through distinct chaperone pathways. Cell 121, 739-748. doi: 10.1016/j.cell.2005.03.024

Meacham, G. C., Patterson, C., Zhang, W., Younger, J. M., and Cyr, D. M. (2001). The Hsc70 co-chaperone CHIP targets immature CFTR for proteasomal degradation. Nat. Cell Biol. 3, 100-105. doi: 10.1038/35050509

Mock, J. Y., Chartron, J. W., Zaslaver, M., Xu, Y., Ye, Y., and Clemons, W. M. Jr. (2015). Bag6 complex contains a minimal tail-anchor-targeting module and a mock BAG domain. Proc. Natl. Acad. Sci. U.S.A. 112, 106-111. doi: 10.1073/pnas.1402745112

Mohamed, B. A., Barakat, A. Z., Held, T., Elkenani, M., Mühlfeld, C., Männer, J., et al. (2014). Respiratory distress and early neonatal lethality in hspa4l/hspa4 double-mutant mice. Am. J. Respir. Cell. Mol. Biol. 50, 817-824. doi: $10.1165 / \mathrm{rcmb} .2013-0132 \mathrm{OC}$

Moro, F., and Muga, A. (2006). Thermal adaptation of the yeast mitochondrial Hsp70 system is regulated by the reversible unfolding of its nucleotide exchange factor. J. Mol. Biol. 358, 1367-1377. doi: 10.1016/j.jmb.2006.03.027

Nakamura, J., Fujimoto, M., Yasuda, K., Takeda, K., Akira, S., Hatayama, T., et al. (2008). Targeted disruption of Hsp110/105 gene protects against ischemic stress. Stroke 39, 2853-2859. doi: 10.1161/STROKEAHA.107.506188

Nelson, G. M., Prapapanich, V., Carrigan, P. E., Roberts, P. J., Riggs, D. L., and Smith, D. F. (2004). The heat shock protein 70 cochaperone hip enhances functional maturation of glucocorticoid receptor. Mol. Endocrinol. 18, 1620-1630. doi: 10.1210/me.2004-0054

Norton, N., Li, D., Rieder, M. J., Siegfried, J. D., Rampersaud, E., Züchner, S., et al. (2011). Genome-wide studies of copy number variation and exome sequencing identify rare variants in BAG3 as a cause of dilated cardiomyopathy. Am. J. Hum. Genet. 88, 273-282. doi: 10.1016/j.ajhg.2011.01.016

Oh, H. J., Chen, X., and Subjeck, J. R. (1997). Hsp110 protects heat-denatured proteins and confers cellular thermoresistance. J. Biol. Chem. 272, 31636-31640. doi: 10.1074/jbc.272.50.31636

Oh, H. J., Easton, D., Murawski, M., Kaneko, Y., and Subjeck, J. R. (1999). The chaperoning activity of hsp110. Identification of functional domains by use of targeted deletions. J. Biol. Chem. 274, 15712-15718. doi: 10.1074/jbc.274.22.15712

Page, R. C., Xu, Z., Amick, J., Nix, J. C., and Misra, S. (2012). Crystallization and preliminary X-ray crystallographic analysis of the Bag2 amino-terminal domain from Mus musculus. Acta Crystallogr. Sect. F Struct. Biol. Cryst. Commun. 68, 647-651. doi: 10.1107/S1744309112013267

Papsdorf, K., Sacherl, J., and Richter, K. (2014). The balanced regulation of Hsc70 by DNJ-13 and UNC-23 is required for muscle functionality. J. Biol. Chem. 289, 25250-25261. doi: 10.1074/jbc.M114.565234

Polier, S., Dragovic, Z., Hartl, F. U., and Brache, A. (2008). Structural basis for the cooperation of Hsp70 and Hsp110 chaperones in protein folding. Cell 133, 1068-1079. doi: 10.1016/j.cell.2008.05.022

Polier, S., Hartl, F. U., and Bracher, A. (2010). Interaction of the Hsp110 molecular chaperones from S. cerevisiae with substrate protein. J. Mol. Biol. 401, 696-707. doi: 10.1016/j.jmb.2010.07.004

Rampelt, H., Kirstein-Miles, J., Nillegoda, N. B., Chi, K., Scholz, S. R., Morimoto, R. I., et al. (2012). Metazoan Hsp70 machines use Hsp110 to power protein disaggregation. EMBO J. 31, 4221-4235. doi: 10.1038/emboj. 2012.264

Rauch, J. N., and Gestwicki, J. E. (2014). Binding of human nucleotide exchange factors to heat shock protein 70 (Hsp70) generates functionally distinct complexes in vitro. J. Biol. Chem. 289, 1402-1414. doi: 10.1074/jbc.M113. 521997

Raviol, H., Sadlish, H., Rodriguez, F., Mayer, M. P., and Bukau, B. (2006). Chaperone network in the yeast cytosol: Hsp110 is revealed as an Hsp70 nucleotide exchange factor. EMBO J. 25, 2510-2518. doi: 10.1038/sj.emboj.7601139

Rogon, C., Ulbricht, A., Hesse, M., Alberti, S., Vijayaraj, P., Best, D., et al. (2014). HSP70-binding protein HSPBP1 regulates chaperone expression at a posttranslational level and is essential for spermatogenesis. Mol. Biol. Cell 25, 2260-2271. doi: 10.1091/mbc.E14-02-0742

Saxena, A., Banasavadi-Siddegowda, Y. K., Fan, Y., Bhattacharya, S., Roy, G., Giovannucci, D. R., et al. (2012). Human heat shock protein $105 / 110 \mathrm{kDa}$ (Hsp105/110) regulates biogenesis and quality control of misfolded cystic fibrosis transmembrane conductance regulator at multiple levels. J. Biol. Chem. 287, 19158-19170. doi: 10.1074/jbc.M111.297580

Schuermann, J. P., Jiang, J., Cuellar, J., Llorca, O., Wang, L., Gimenez, L. E., et al. (2008). Structure of the Hsp110:Hsc70 nucleotide exchange machine. Mol. Cell 31, 232-243. doi: 10.1016/j.molcel.2008.05.006

Selcen, D., Muntoni, F., Burton, B. K., Pegoraro, E., Sewry, C., Bite, A. V., et al. (2009). Mutation in BAG3 causes severe dominant childhood muscular dystrophy. Ann. Neurol. 65, 83-89. doi: 10.1002/ana.21553. doi: 10.1002/ana.21553

Senderek, J., Krieger, M., Stendel, C., Bergmann, C., Moser, M., Breitbach-Faller, N., et al. (2005). Mutations in SIL1 cause Marinesco-Sjogren syndrome, a cerebellar ataxia with cataract and myopathy. Nat. Genet. 37, 1312-1314. doi: $10.1038 / \mathrm{ng} 1678$

Shaner, L., Trott, A., Goeckeler, J. L., Brodsky, J. L., and Morano, K. A. (2004). The function of the yeast molecular chaperone Sse1 is mechanistically distinct from the closely related hsp70 family. J. Biol. Chem. 279, 21992-22001. doi: 10.1074/jbc.M313739200

Shomura, Y., Dragovic, Z., Chang, H. C., Tzvetkov, N., Young, J. C., Brodsky, J. L., et al. (2005). Regulation of Hsp70 function by HspBP1: Structural analysis reveals an alternate mechanism for Hsp70 nucleotide exchange. Mol. Cell 17, 367-379. doi: 10.1016/j.molcel.2004.12.023

Shorter, J. (2011). The mammalian disaggregase machinery: Hsp110 synergizes with Hsp70 and Hsp40 to catalyze protein disaggregation and reactivation in a cell-free system. PLoS ONE 6:e26319. doi: 10.1371/journal.pone.0026319

Sondermann, H., Scheufler, C., Schneider, C., Höhfeld, J., Hartl, F. U., and Moarefi, I. (2001). Structure of a Bag/Hsc70 complex: convergent functional evolution of Hsp70 nucleotide exchange factors. Science 291, 1553-1557. doi: 10.1126/science. 1057268

Steel, G. J., Fullerton, D. M., Tyson, J. R., and Stirling, C. J. (2004). Coordinated activation of Hsp70 chaperones. Science 303, 98-101. doi: 10.1126/science. 1092287

Takayama, S., and Reed, J. C. (2001). Molecular chaperone targeting and regulation by BAG family proteins. Nat. Cell Biol. 3, E237-E241. doi: 10.1038/ 35082637

Tyson, J. R., and Stirling, C. J. (2000). LHS1 and SIL1 provide a lumenal function that is essential for protein translocation into the endoplasmic reticulum. EMBO J. 19, 6440-6452. doi: 10.1093/emboj/19.23.6440

Tzankov, S., Wong, M. J., Shi, K., Nassif, C., and Young, J. C. (2008). Functional divergence between co-chaperones of Hsc70. J. Biol. Chem. 283, 27100-27109. doi: 10.1074/jbc.M803923200 
Ulbricht, A., Eppler, F. J., Tapia, V. E., Van Der Ven, P. F., Hampe, N., Hersch, N., et al. (2013). Cellular mechanotransduction relies on tensioninduced and chaperone-assisted autophagy. Curr. Biol. 23, 430-435. doi: 10.1016/j.cub.2013.01.064

Ung, P. M., Thompson, A. D., Chang, L., Gestwicki, J. E., and Carlson, H. A. (2013). Identification of key hinge residues important for nucleotidedependent allostery in E. coli Hsp70/DnaK. PLoS Comput. Biol. 9:e1003279. doi: 10.1371/journal.pcbi.1003279

Verghese, J., and Morano, K. A. (2012). A lysine-rich region within fungal BAG domain-containing proteins mediates a novel association with ribosomes. Eukaryot. Cell 11, 1003-1011. doi: 10.1128/EC. 00146-12

Wang, A. M., Miyata, Y., Klinedinst, S., Peng, H. M., Chua, J. P., Komiyama, T., et al. (2013). Activation of Hsp70 reduces neurotoxicity by promoting polyglutamine protein degradation. Nat. Chem. Biol. 9, 112-118. doi: 10.1038/nchembio. 1140

Wang, J., Farr, G. W., Zeiss, C. J., Rodriguez-Gil, D. J., Wilson, J. H., Furtak, K., et al. (2009). Progressive aggregation despite chaperone associations of a mutant SOD1-YFP in transgenic mice that develop ALS. Proc. Natl. Acad. Sci. U.S.A. 106, 1392-1397. doi: 10.1073/pnas.0813045106

Wilbanks, S. M., and Mckay, D. B. (1995). How potassium affects the activity of the molecular chaperone Hsc70. II. Potassium binds specifically in the ATPase active site. J. Biol. Chem. 270, 2251-2257. doi: 10.1074/jbc.270. 5.2251

Willmund, F., Mühlhaus, T., Wojciechowska, M., and Schroda, M. (2007). The $\mathrm{NH} 2$-terminal domain of the chloroplast GrpE homolog CGE1 is required for dimerization and cochaperone function in vivo. J. Biol. Chem. 282, 11317-11328. doi: 10.1074/jbc.M608854200

Xu, X., Sarbeng, E. B., Vorvis, C., Kumar, D. P., Zhou, L., and Liu, Q. (2012). Unique peptide substrate binding properties of $110-\mathrm{kDa}$ heat-shock protein (Hsp110) determine its distinct chaperone activity. J. Biol. Chem. 287, 5661-5672. doi: 10.1074/jbc.M111.275057
Xu, Z., Graham, K., Foote, M., Liang, F., Rizkallah, R., Hurt, M., et al. (2013). 14-3-3 protein targets misfolded chaperone-associated proteins to aggresomes. J. Cell. Sci. 126, 4173-4186. doi: $10.1242 /$ jcs. 126102

Xu, Z., Page, R. C., Gomes, M. M., Kohli, E., Nix, J. C., Herr, A. B., et al. (2008). Structural basis of nucleotide exchange and client binding by the Hsp70 cochaperone Bag2. Nat. Struct. Mol. Biol. 15, 1309-1317. doi: 10.1038/ nsmb.1518

Yam, A. Y., Albanese, V., Lin, H. T., and Frydman, J. (2005). HSP110 cooperates with different cytosolic HSP70 systems in a pathway for de novo folding. J. Biol. Chem. 280, 41252-41261. doi: 10.1074/jbc.M503615200

Yan, M., Li, J., and Sha, B. (2011). Structural analysis of the Sill-Bip complex reveals the mechanism for Sill to function as a nucleotide-exchange factor. Biochem. $J$. 438, 447-455. doi: 10.1042/BJ20110500

Zhao, L., Longo-Guess, C., Harris, B. S., Lee, J. W., and Ackerman, S. L. (2005). Protein accumulation and neurodegeneration in the woozy mutant mouse is caused by disruption of SIL1, a cochaperone of BiP. Nat. Genet. 37, 974-979. doi: $10.1038 /$ ng 1620

Zimmermann, R., Eyrisch, S., Ahmad, M., and Helms, V. (2011). Protein translocation across the ER membrane. Biochim. Biophys. Acta 1808, 912-924. doi: 10.1016/j.bbamem.2010.06.015

Conflict of Interest Statement: The authors declare that the research was conducted in the absence of any commercial or financial relationships that could be construed as a potential conflict of interest.

Copyright (c) 2015 Bracher and Verghese. This is an open-access article distributed under the terms of the Creative Commons Attribution License (CC BY). The use, distribution or reproduction in other forums is permitted, provided the original author(s) or licensor are credited and that the original publication in this journal is cited, in accordance with accepted academic practice. No use, distribution or reproduction is permitted which does not comply with these terms. 